\title{
KEEP FEELING FASCINATION: DESIGN AND OPEN ENQUIRY
}


All too often, we say what we hear others say. We think what we're told that we think. We see what we're permitted to see. Worse! We see what we're told that we see.-Octavia Butler '즌..

We live in times when we are reminded daily of the possible collapse of environmental, social, political and economic systems. At the same time, there is a feeling that the world has become exceedingly complex: although we are able to access vast quantities of information, it seems increasingly difficult to comprehend the interconnectedness of our lives. This feeling of a growing complexity has, however, accompanied humankind throughout history. With our ongoing externalisation of human capacities, we have been trying to keep up with the technologies that were originally meant to unburden us. And although our desire for wealth and comfort exceeds the availability of natural resources, we have been striving to multiply our systems of production, creating precarious networks of labour. We seek knowledge, but become overwhelmed by the amassment of data and its infinite linkages. The current remedy for perceived complexity is to have computing technologies quantify every aspect of our lives. Quantification promises control-but also impacts our view of the world: “The issue may not be so much the extent to which the world is unpredictable as the extent to which we are disturbed by its unpredictability. [...] the use of technology has not, in fact, made the 
world a more predictable or safe place. But perhaps a more technologically oriented way of thinking has made us more averse to unpredictability and its consequent risk." 改

In the field of design, the aforementioned aversion to risk finds expression in the idea that design is a process of problem-solving. This idea suggests that complex issues can be reduced to distinct, neatly compartmentalised problems for which an appropriate method must be found, and whose application will result in a fitting solution. Put more provocatively, this means that designers frame problems as design problems that necessitate design methods and produce design solutions. And even though many parts of the design process escape quantification, the narrative of problem-solving suggests control, with volatile aspects of the process ideally receding into the background. By solving the problem, the problem disappears ... because it has been replaced by the solution. This latter notion, however, brings with it conceptual dilemmas, especially when tackling complex issues. For instance: when exactly has awareness for an issue been achieved? When exactly has society been changed?

The unknown, [...] the unforetold, the unproven, that is what life is based on. Ignorance is the ground of thought. Unproof is the ground of action. [...] The only thing that makes life possible is permanent, intolerable uncertainty; not knowing what comes next.-Ursula $K$. LeGuin 산자 
The desire to create a material solution to counter a problem runs deep, also within the field of visual communication. However, it sits uncomfortably within this notion, as the true skill within visual communication consists not of dealing with technical aspects of producing the solution, but rather with making sense of a particular circumstance or topic and allowing others access to it. In other words, visual designers should not aim to solve problems, but to engage with an issue or phenomenon in order to attain a perspective on it and share that perspective with others.

Making sense of an issue or phenomenon is a process that may be supported by common-sense heuristics and expert knowledge where available, but it does not benefit from prescription. Making sense of something resists being simplified and cannot be defined from $A$ to $Z$. It is, rather, a process that requires openness and curiosity, and the courage to deal with the pitfalls and uncertainties that will necessarily emerge. It requires a distancing from prior assumptions about the issue, and a bracketing of prior knowledge-two methodological requirements that run counter to the desire to solve problems quickly and efficiently. An unlearning of this kind may lead to worry and uncertainty, since a distancing from personal, disciplinary or professional assumptions calls into question the role that society attributes to the designer, and the role and position one has acquired for oneself through years of education and practice. Without unlearning and bracketing out knowledge, however, other possible views on the issue cannot surface. They are, instead, constantly over- 
shadowed by the conviction of designers that they have fully understood all that there is to understand about the issue, and that they know what is best for the intended communication recipient. Unlearning furthermore shifts attention to forms of knowledge that are usually not prioritised. It allows for an enquiry based on hunches, gut feelings, not-fully-resolved thoughts or contradictions. Encountering uncertainty and complexity becomes central, as does the search for ways to make both productive for the process of enquiry. The shift implied is also an ontological one towards perceiving the world, which for humankind is always a social world, as a world in process. Open enquiry traces this process; it learns from world-making and intervenes in it.

This is why a design enquiry should not be misunderstood as a gathering of facts or bits of data to justify the development of a product that the designer had decided to make even before they started the enquiry. Instead, it should be taken to denote a process of thinking oneself through an issue by means of observing, reflecting and prospective designing. The mere fact that the sense-making process cannot be prescribed or contained in a universally applicable model does not necessarily indicate that it is arbitrary. Rigour can be fostered in many ways: for instance through attention, criticality, empathy and imagination.

We might try to understand this process further through three concepts: correspondence, presence and trust. Correspondence points to several aspects that are enfolded in the multiple meanings of the term. It is first of all a process of communication, of dialogue; 
secondly, it denotes an engagement and possible alignment between two entities: two things correspond to each other. Sometimes both of these meanings may converge: correspondence in observation, for instance, can mean "a level of mutual involvement, in perception and action, such that observer and observed become all but indistinguishable." ( ()$_{\Lambda_{\lambda}}^{v v^{\prime}}$ Presence, in turn, requires the designer to be fully present, in that they are fully attending to the topic and fully committing themselves intellectually to seeking a new perspective. Presence means avoiding superficial conclusions, as these require only half of one's attention, with the other half focused on realising a pre-set goal on which the actual setting has little effect. Presence also implies paying attention to oneself, with designers allowing themselves to be transformed by the experience of enquiring. The third concept, that of trust, encourages the designer to trust in the enquiry and the process of designing as well as in the human capacity and desire to question well-established concepts in order to gain a new perspective. Trust requires the observer to commit with compassion and openness to any kind of encounter.

We are gorgeously contradictory in our epistemologies.-Elizabeth Alexander $\equiv>^{\prime \prime}$

By shifting perspective and examining the medium through which a newly attained perspective is to be communicated, designers are able 
to reposition themselves in relation to their practice. They produce intersubjective knowledge that is made perceptible and experienceable through designed artefacts, $\frac{\mathrm{VV} / \mathrm{V}}{1 / \times 1 \mathrm{~V}}$ rather than relying solely on written or verbal accounts. These artefacts may, for instance, give form to marginalised knowledge, question conventional paths or methods, or reveal how the knowledge they embody has been constructed. Through this, they allow new possibilities of encountering a topic. They also encourage speculation and critique. They involve others in dialogue, both about the knowledge they claim and the conditions of their making. The following four examples of graduate work from the MA in Visual Communication at Zurich University of the Arts (ZHdK) demonstrate the diversity of approaches that may be taken, as well as their common conceptual grounding in openness and exploration.

"What about you, why are you still here? Well, I am talking to you" by Daniela Gruber is a project that reconsiders the boundaries between what society considers healthy and ill. Autism and autistic behaviour form the focal point of the project, which is supported by interviews and a comprehensive review of the medical literature. In order to attain a new perspective on autism that circumvents medical and social ascriptions, Gruber filtered out aspects of described autistic behaviour, such as the search for recurring patterns, and transformed these into visual experiments. The experiments were to allow her a 
glimpse into a lived experience, albeit constrained by the impossibility of ever accessing or grasping this experience herself (thus she also never suggested that what she was experiencing was comparable to the lived experience of an autist). Openness was crucial for this enquiry-any pre-set ideas about autism would have steered Gruber's own experience of the experiments onto conventional paths. Subsequently, she examined the suitability of the book as a medium for communicating the perspective gained through the experiments. The book was intended to show how she encountered autism, and thus to provide a perspective supplementary to the medical literature and knowledge of parents of autistic children. Through design choices concerning typography, images, dramaturgy and colour, Gruber explored how to allow the reader to encounter the book in correspondence with how she had encountered autistic behaviour. The book reveals her insights, but also escapes direct access - for instance, chapter titles are printed on transparent sheets that are not bound within the remaining pages, which means that when the book is handled, these sheets may fall out and scatter. The fragility implied through material aspects of the book further emphasises the fragility and transmutability of knowledge, and in this case especially, the social dimension of medical diagnosis and categorisation.

"First Impressions" by Elliot Frydenberg consists of a visual essay that is shown via a multi-channel installation, and challenges stereotypical depictions of gay men. Frydenberg attests that although visibility has been fought 


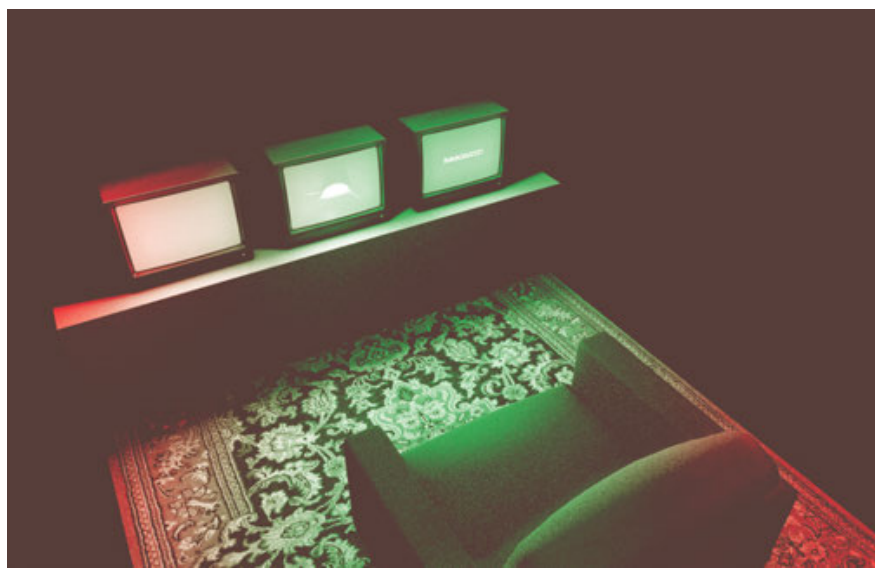

FIG. 1

FIG. 2

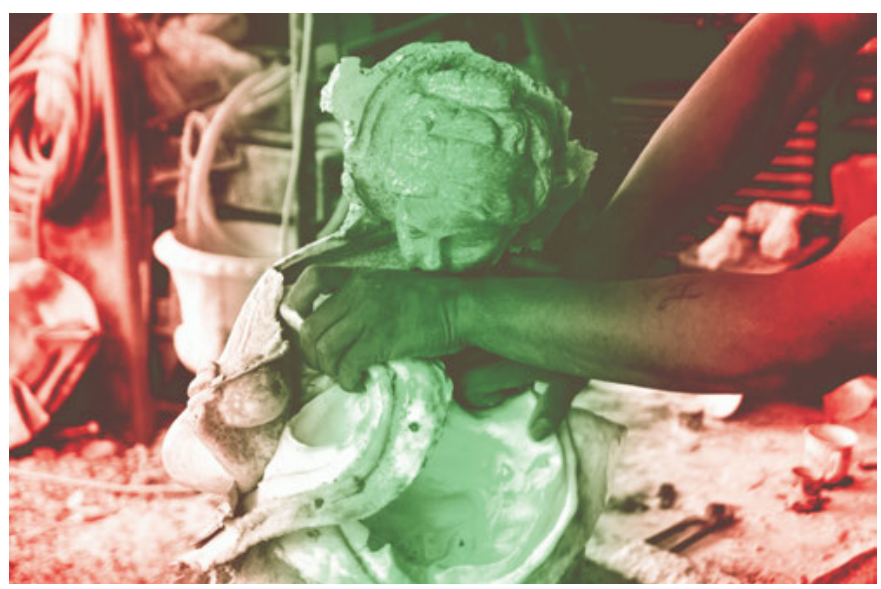

FIG. 3

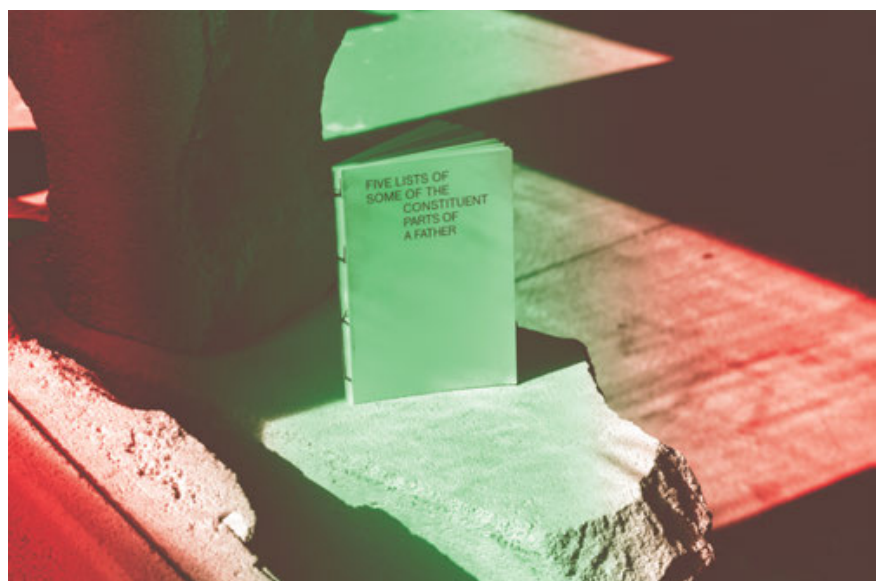


for and achieved in western media, many representations resort to simplistic tropes and gloss over lived experiences. In order to gain a new perspective on these representations, and to diversify the scope of narratives expressing experiences of gay men, Frydenberg took as a starting point the filmic representations he himself had encountered. With the aim of redefining imagery that is tightly linked to memories and emotions as well as the shaping of identity, the project tackled the fundamental question of where to locate alternatives to the visuality set by popular media-quite possibly, in the gaps left by the popular images themselves. by means of joining and juxtaposing selected popular media depictions, Frydenberg disrupts this narrative by introducing textual fragments of a personal narrative, and images of objects that function as indices of others' testimonies and experiences. They render identity not as fixed, but as fluid and adaptive. Media content, fragments and object images encourage the viewer to follow the recurrent shift in perspective that oscillates between proximity and distance, thus creating a methodical link to Frydenberg's approach.

Stereotypical, biased media representations were also the starting point for Reinhard Schmidt's project "Palms on Balconies." This project centres on houses in Romania that are built for representation and possess a particular form of architectural expression. Although such buildings exist around the world and have 
aesthetic similarities, the media in Romania deride the houses as "Roma palaces," thus fuelling public resentment towards Roma people. To gain a new perspective on this architecture, Schmidt employed methods of visual ethnography as well as a systematised editorial process. On several visits to Romanian cities that have recently seen expansion through representational houses, photography was the main means of visually exploring and analysing the relationship between the houses, their characteristics and their environment. Informal interviews with builders and house owners supplemented the visual material gathered, and provided an additional means for understanding the intentions of those involved in planning and building the houses. This open enquiry led to the insight that many characteristic and quite spectacular architectonic elements were decided upon not only by the house owners (as some of them lived in other countries), but by the personal preferences of the builders, the availability of materials on the market, or references to other prominent historical or contemporary buildings. In a second phase, Schmidt explored how to communicate the insights and perspectives he had gained. The resulting book works through architectural typologies and at the same time leaves space for accounts and views that are hidden at first sight. The book is not a solution. Rather, its contingency leads to questions of epistemology: how do we perceive and gain knowledge about the built environment? What importance do we attribute to images in such an enquiry? What insights emerge from assembling and establishing relationships between images? 
With her project "Towards a narrative exploration of experiences," Annamaria Kozma set out from the premise that narratives shape our understanding of the world and play an important role in conveying our experiences. Based on extensive research in philosophy, psychology and literary theory, Kozma aimed to inquire more specifically how certain forms of narrative correspond to or influence the experiences they are meant to mediate. In order to answer this question, she chose an experience that is experienced by many: having a father. Using her relationship with her own father as an example, she experimentally developed five narrative formats and thus five perspectives that demonstrate multiple ways in which such experiences may be thought about, viewed or told. By repeatedly encountering the material she had gathered (family photographs, for instance), and by closely examining how to give visual form to aspects of lived experiences that may escape verbalisation, Kozma attained a level of presence and correspondence with the phenomenon, i.e., a committed, full attention to the structure and meaning of the experience studied. Physically manifest through five publications, the perspectives enter into a dialogue and again allow new insights. Their interplay both shows the influence of the narrative on the experience and questions it. Readers are encouraged to enter into this dialogue, to engage with it, and to connect their own experience to that which is presented.

"Image" increasingly rules the realm of shaping, sometimes becoming, often contaminating, knowledge. Provoking language or eclips- 
ing it, an image can determine not only what we know and feel but also what we believe is worth knowing about what we feel.-Toni Morrison

Abandoning the goal of problem-solving generates uncertainty about the result of the design process. However, this assumes that the preferred result of the design process is a product. An open enquiry within visual communication-one that is set in motion by observation, unlearning and prospective thinking-instead leads to a different perspective, a new possibility that in turn engenders new ways of seeing, thinking and doing. Most importantly, however, it leads to a transformation of the designers themselves, who by being deeply and fully involved with the issue or phenomenon and by learning from it cannot help but change. 



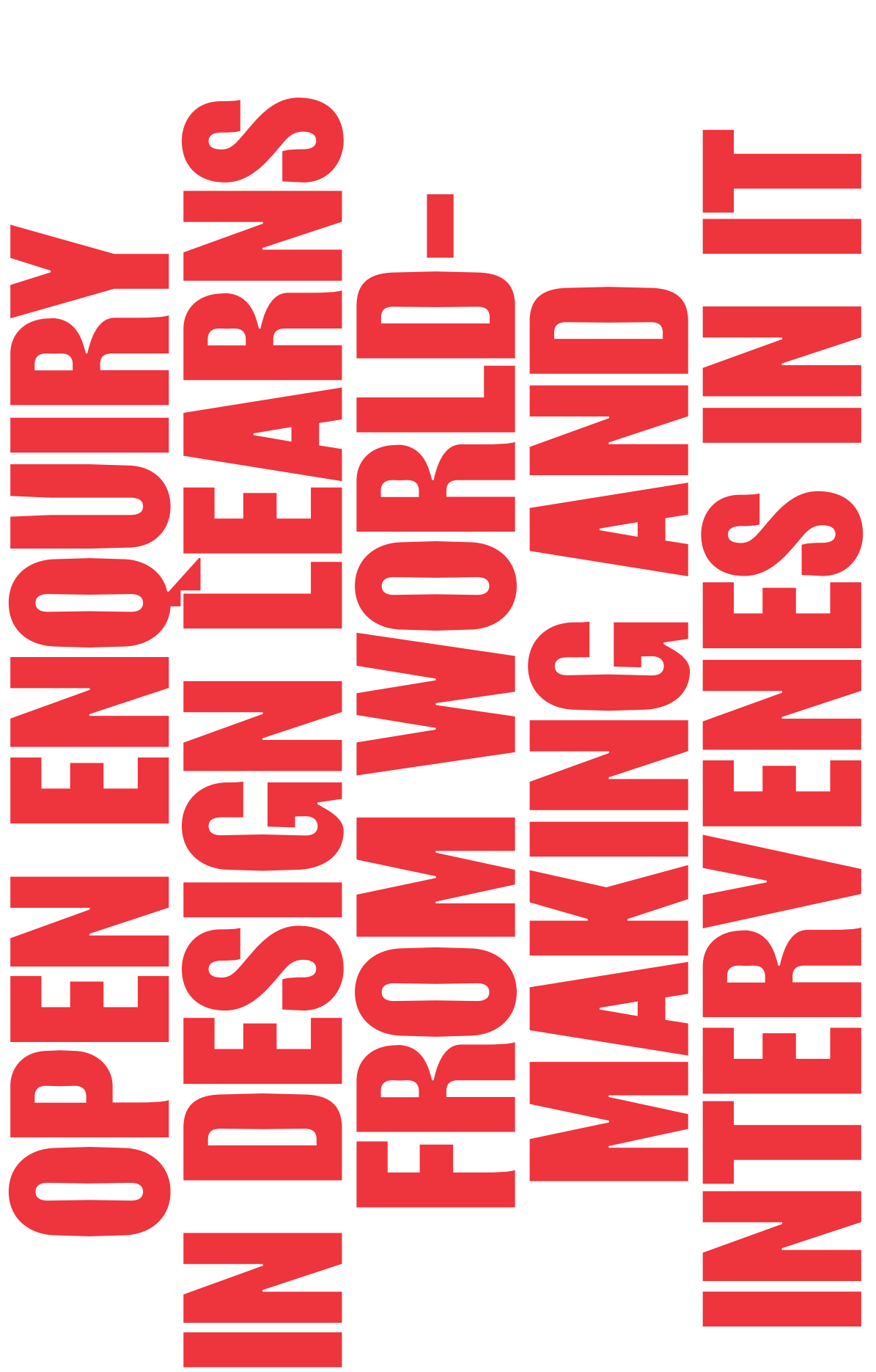

WORLD

P.XXI

P.XXIV

P. XXX

P.XXXI

P. 58

P. 64

P. 77

P. 81

P. 110

P. 111

P. 112

P. 113

P. 114

P. 115

P. 126

P. 129

P. 136

P. 142

P. 147

P. 209

P. 212

P. 222

P. 223

P. 238

P. 243

P. 251

P. 254

P. 262

P. 264

P. 268

P. 272

P. 320

P. 335

P. 337

P. 370

P. 372

P. 422

P. 424

P. 430

P. 432

P. 442

P. 443 
